\title{
Decreased Meiotic Intergenic Recombination and Increased Meiosis I Nondisjunction in exo1 Mutants of Saccharomyces cerevisiae
}

\author{
David T. Kirkpatrick, ${ }^{*, 1}$ John R. Ferguson, ${ }^{\dagger}$ Thomas D. Petes* and Lorraine S. Symington ${ }^{\dagger}$ \\ *Department of Biology and Curriculum in Genetics and Molecular Biology, University of North Carolina, Chapel Hill, \\ North Carolina 27599-3280 and ${ }^{\dagger}$ Department of Microbiology, Columbia University College of \\ Physicians and Surgeons, New York, New York 10032 \\ Manuscript received July 23, 1999 \\ Accepted for publication September 6, 2000
}

\begin{abstract}
Exonuclease I was originally identified as a $5^{\prime} \rightarrow 3^{\prime}$ deoxyribonuclease present in fractionated extracts of Schizosaccharomyces pombe and Saccharomyces cerevisiae. Genetic analysis of exo1 mutants of both yeasts revealed no major defect in meiosis, suggesting that exonuclease I is unlikely to be the primary activity that processes meiosis-specific double-strand breaks (DSBs). We report here that exo1 mutants of $S$. cerevisiae exhibit subtle but complex defects in meiosis. Diploids containing a homozygous deletion of EXO1 show decreased spore viability associated with an increase in meiosis I nondisjunction, while intergenic recombination is reduced about twofold. Exolp functions in the same pathway as Msh5p for intergenic recombination. The length of heteroduplex tracts within the HIS4 gene is unaffected by the exol mutation. These results suggest that Exolp is unlikely to play a major role in processing DSBs to form single-stranded tails at HIS4, but instead appears to promote crossing over to ensure disjunction of homologous chromosomes. In addition, our data indicate that exonuclease I may have a minor role in the correction of large DNA mismatches that occur in heteroduplex DNA during meiotic recombination at the HIS4 locus.
\end{abstract}

$I^{\mathrm{N}}$ Saccharomyces cerevisiae, meiotic recombination is initiated by double-strand breaks (DSBs) that are subsequently processed to form 3 ' single-stranded tails (CAO et al. 1990; Sun et al. 1991). Spo11p is an atypical type II topoisomerase that is thought to catalyze DSB formation (KEENEY et al. 1997), but the identity of the nuclease(s) that processes the breaks to form singlestranded tails remains unknown. The processing reaction is thought to require the activity of a $5^{\prime} \rightarrow 3^{\prime}$ double-stranded exonuclease, a $5^{\prime} \rightarrow 3^{\prime}$ single-stranded exonuclease in conjunction with a DNA helicase, or a single-stranded endonuclease, again working with a DNA helicase. In some proposed models, the Mre11/ $\operatorname{Rad} 50 / \mathrm{Xrs} 2$ complex participates in the removal of Spo11p from DSB sites in meiosis and might also participate in the removal of the $5^{\prime}$ strand (FURUse et al. 1998; Usu i et al. 1998; Moreau et al. 1999). However, the in vitro exonuclease activities of yeast Mre11p and the human Mre11 complex are of the opposite polarity to that predicted for the processing reaction (FURUSE et al. 1998; Trujillo et al. 1998; Usui et al. 1998). Although the endonuclease activity of Mre11p might be sufficient to remove Spo11p and the $5^{\prime}$ strand, another possibility

Corresponding author: Lorraine S. Symington, Department of Microbiology and Institute of Cancer Research, Columbia University, 701 W. 168th St., New York, NY 10032. E-mail: 1ss5@columbia.edu

${ }^{1}$ Present address: Department of Genetics, Cell Biology, and Development, University of Minnesota, 250 Biological Sciences Bldg., 1445 Gortner Ave., St. Paul, MN 55108. is that the $5^{\prime}$ strand is removed by the sequential action of Mre11p and a different nuclease.

The resulting 3' single-stranded tailed molecules participate in homologous pairing and strand invasion of a homologous duplex to form heteroduplex DNA. The length of heteroduplex DNA at the ARG4 locus is dictated by the extent of degradation of the $5^{\prime}$ strand (SuN et al. 1991). DNA mismatches present in heteroduplex DNA are repaired by the mismatch correction system giving rise to gene conversion (6:2 or 2:6 segregation of markers) or restoration to the parental markers (4:4 segregation of markers). Failure to repair results in postmeiotic segregation of markers to yield $5: 3$ or 3:5 segregations, manifested as sectored spore colonies in $\mathrm{S}$. cerevisiae. Although single base pair (except $\mathrm{C} / \mathrm{C}$ ) and small insertion/deletion mismatches are efficiently repaired by the mismatch repair system, palindromic insertions are refractory to repair (NAG et al. 1989). Hence, palindromic insertions can be used to monitor heteroduplex DNA formation without the complication of effects on meiotic recombination and sporulation caused by defects in the mismatch repair system. At the HIS4 locus in $S$. cerevisiae, heteroduplex formation in meiosis is initiated at the $5^{\prime}$ end of the gene and often extends to the $3^{\prime}$ end, a distance of $\sim 2.5 \mathrm{~kb}$ (PorTer et al. 1993).

Exonuclease I was originally characterized because the biochemical activity suggested that it might play a role in processing DSBs in vivo (SZANKASI and SMITH 1992; HuAng and Symington 1993; Fiorentini et al. 1997). The exo1 gene of Schizosaccharomyces pombe is transcriptionally induced during meiosis, suggesting a role in 
meiotic DNA metabolism (Szankasi and SMith 1995). The observations that exo1 mutants are radiation resistant, proficient at mating-type switching, and have wildtype levels of sporulation argue against the possibility that exonuclease I is the primary DSB-processing activity (Szankasi and Smith 1995; Fiorentini et al. 1997). Instead, the elevated rates of spontaneous mutation observed in exo1 strains and the physical interaction between Exo1p and Msh2p are more consistent with a role for Exolp in mismatch repair in mitotic cells. TsubouCHI and Ogawa (2000) isolated EXO1 as a high-copy suppressor of the methyl methanesulfonate sensitivity caused by mutations in MRE11, RAD50, and XRS2. They also show that EXO1 is induced in meiosis; processing of double-strand breaks is reduced in an exol dmc1 strain; the level of crossing over, but not the frequency of gene conversion, is reduced by the exo1 mutation; and Exolp and Msh4p act in the same pathway for intergenic recombination. KHAZANEHDARI and BORTS (2000) also demonstrate that Exo1p and Msh4p act in the same pathway for crossing over but act in different pathways for spore viability. They show that the exo1 mutation confers a high rate of meiosis I nondisjunction and that the level of gene conversion in the mutant is reduced at some but not all alleles examined.

Below we report that Exolp has several meiotic functions. Strains with the exo1 mutation have decreased spore viability and intergenic recombination and have elevated levels of chromosome nondisjunction. Genetic data argue that Exo1p acts in the same pathway as Msh5p to generate intergenic recombinants, but in overlapping pathways for spore viability. The extent of heteroduplex formation is not affected by the exo1 mutation, indicating that exonuclease I does not have an essential role in processing of double-strand breaks to generate long $3^{\prime}$ single-stranded DNA molecules for strand invasion during meiotic recombination. In addition, the patterns of aberrant segregation observed in exol strains suggest that Exolp functions in the repair of the DNA mismatches that lead to gene conversion events at some loci. These results are consistent with Exolp having complex roles in both meiotic intergenic recombination and mismatch repair.

\section{MATERIALS AND METHODS}

Media and genetic procedures: With the exceptions noted below, standard protocols and media were used (SHERman et al. 1986). Sporulation plates contained $1 \%$ potassium acetate, $0.1 \%$ yeast extract, $0.05 \%$ glucose, $6 \mu \mathrm{g} / \mathrm{ml}$ adenine, and $2 \%$ agar. For studies on the frequencies of crossovers and aberrant segregation, diploids were sporulated at $18^{\circ}$ and dissected onto plates containing rich growth medium. After colonies formed, they were replica plated to various omission media to score segregation of heterozygous markers. Postmeiotic segregation events at the HIS4 locus were detected as sectored $\mathrm{His}^{+} / \mathrm{His}^{-}$colonies (Detloff et al. 1992). Nomenclature for aberrant segregation events follows the standard set for eightspored fungi-for segregation of a heterozygous allele A/a, Men- delian segregation is designated 4A:4a, while a gene conversion event is $6 \mathrm{~A}: 2 \mathrm{a}$ or $2 \mathrm{~A}: 6 \mathrm{a}$ and single postmeiotic segregation events (one sectored colony) are 5A:3a and 3A:5a.

Studies on spore viability were done in two different genetic backgrounds. Diploids in the AS4/AS13 genetic background (described below) were sporulated at $18^{\circ}$. Diploids in the W303 background were sporulated at $30^{\circ}$. Following matings of the haploids to generate diploids, these strains were grown for either 15 or 60 generations prior to sporulation. The mating phenotype of germinated spores was determined by replica plating dissection plates to lawns of mating-type tester strains MCY14 and SJR13, followed by selection for diploids on minimal medium. Spores that failed to mate to the two tester strains were considered to be nonmaters due to missegregation of chromosome III.

Strains: Diploid strains used in the analysis of recombination were constructed by mating haploid strains derived from AS4 (MATo trp1-1 arg4-17 tyr7-1 ade6 ura3) or AS13 (MATa leu2Bst ade6 ura3 rme1) (Stapleton and Petes 1991) or from published derivatives of these strains. The haploid DTK299 was made from AS4 by one-step gene replacement using a PCR-generated DNA fragment designed to replace the EXO1 gene with the URA3 gene. The PCR fragment was generated by using the primers ( $5^{\prime}$ AAAGGAGCTCGAAAAAACTGAA AGGCGTAGAAAGGAATGGGTATCCAAGGTgattgtactgagagt gcacc and 5' CCTCCGATATGAAACGTGCAGTACTTAACT TTTATTTACCTTTATAAACAAATTGGGgggctgtgcggtattcac accg) to amplify the URA3 gene from the plasmid pRS306 (SIKORski and Hieter 1989). Capital letters represent EXO1 sequences and are identical to those used previously to construct the exo1::HIS3 allele (Fiorentini et al. 1997); the small letters represent sequences flanking URA3. Other strains with the exo1 mutation were constructed in the same way including: DTK300, derived from DNY24 [AS13 but his4-lopd, (NAG et al. 1989)]; LSY612, derived from DNY16 [AS13 but his4-B2 (NAG et al. 1989)]; LSY613, derived from DNY25 [AS13 but his4-lopc (NAG et al. 1989)]; and LSY611, derived from PD98 [AS13 but his4-3133 (Detloff et al. 1992) ]. MSH5 was disrupted in DNY24, creating DTK492, using a PCR-generated DNA fragment from the primers $\left(5^{\prime}\right.$ CAACTCATTCAAAATAACT TACTCATTCATATACTGCCACCAAATGGAATcgtacgctgcag gtcgac and 5' TTATTAACTTAAATATGTTACAGGTGGGCG TTTTTTTATTCTTTGATATAatgatgaattcgagctcg) to amplify the KanMX4 cassette from pFA6-kanMX4 (WACH et al. 1994); transformants were selected by resistance to geneticin. Capital letters in the primers represent $M S H 5$ sequences, while small letters represent pFA6-kanMX4 sequences. DTK492 was crossed with AS4 and transformed by a one-step transformation using the msh5::URA3 plasmid pNH190-11 (HoLlingsWORTH et al. 1995), selecting for $\mathrm{Ura}^{+}$geneticin-resistant transformants to create the diploid DTK498. MSH5 was disrupted in a Ura ${ }^{-}$derivative of DTK299 using pNH190-11, generating DTK502. EXO1 was disrupted in DTK492 using the exo1::URA3 primers described above, creating DTK504. Diploid strains were constructed by mating: DTK308 (DTK299 × DTK300), DTK332 (DTK299 × LSY612), DTK333 (DTK299 × LSY613), DTK335 (DTK299 $\times$ LSY611), and DTK505 (DTK502 $\times$ DTK504). Except for the exo1 and/or msh 5 mutations, strains DTK332, DTK333, and DTK335 are isogenic with strains DNY16, DNY26, and PD98, while DTK308, DTK498, and DTK505 are isogenic with DNY27 (NAG et al. 1989; DETLOFF et al. 1992). The diploid strain DTK347 is also isogenic with the diploid DNY26 (NAG et al. 1989), except for a homozygous insertion of a 60-bp simple repetitive sequence (CCCGNN) $)_{12}$ that replaces transcription factor binding sites located upstream of HIS4 (KIRKPATRICK et al. 1999).

Since diploids derived from AS4 and AS13 exhibit lower spore viability than most other laboratory strains, we also used 
TABLE 1

Viability of spores from wild-type and exo1 diploids (W303 genetic background)

\begin{tabular}{|c|c|c|c|c|c|c|c|c|c|}
\hline \multirow[b]{2}{*}{ Strain } & \multirow[b]{2}{*}{ EXO1 } & \multirow[b]{2}{*}{$\begin{array}{c}\text { No. of } \\
\text { generations }^{b}\end{array}$} & \multirow[b]{2}{*}{$\begin{array}{l}\text { No. of } \\
\text { tetrads }\end{array}$} & \multicolumn{6}{|c|}{$\%$ tetrads with indicated spore viability pattern (nos. of tetrads) ${ }^{a}$} \\
\hline & & & & 4:0 & $3: 1$ & $2: 2$ & $1: 3$ & $0: 4$ & $\begin{array}{l}\% \text { spore } \\
\text { viability }\end{array}$ \\
\hline W303 & $+/+$ & 15 & 1012 & $82(833)$ & $14(138)$ & $3(30)$ & $0.5(5)$ & $0.5(6)$ & 94 \\
\hline LSY529a & $-/-$ & 15 & 492 & $72(355)$ & $13(63)$ & $9(45)$ & $1(6)$ & $5(23)$ & 87 \\
\hline LSY529b & $-/-$ & 15 & 513 & 74 (379) & $11(56)$ & $9(48)$ & $2(8)$ & $4(22)$ & 87 \\
\hline \multicolumn{10}{|l|}{$\mathrm{LSY}^{2} 2^{c}$} \\
\hline (summed) & $-/-$ & 15 & 1005 & $73(734)$ & 12 (119) & $9(93)$ & $1(14)$ & $5(45)$ & 87 \\
\hline W303 & $+/+$ & 60 & 197 & 77 (152) & $8(16)$ & $10(19)$ & $3(6)$ & $2(4)$ & 89 \\
\hline LSY529 & $-/-$ & 60 & 213 & $66(140)$ & $9(18)$ & $13(28)$ & $2(5)$ & $10(22)$ & 79 \\
\hline
\end{tabular}

${ }^{a}$ Strains were sporulated and four-spored tetrads were dissected. The number of viable spores is shown, followed by the number of inviable spores. For example, the 4:0 class represents tetrads with four viable and no inviable spores.

${ }^{b}$ Following construction of the diploid strains, the cells were grown for 15 or 60 generations prior to meiosis.

' Summed data from LSY529a (exo1:HIS3/exo1:HIS3) and LSY529b (exo1:URA3/exo1:HIS3) diploids. No statistical difference was noted between LSY529a and LSY529b, but the heterozygous exo1 insertion in LSY529b allows monitoring of nondisjunction events involving chromosome $X V$ (see text).

W303 derivatives to measure spore viability. Strains W303-1A (MATa leu2-3, 112 trp1-1 can1-100 ura3-1 ade2-1 his3-11, 15) and

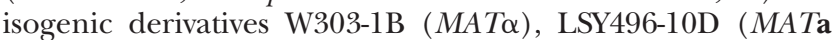
exo1::HIS3), and LSY496-20A (MAT $\alpha$ exo1::HIS3) were described previously (FIORENTINi et al. 1997). LSY625 (MATa exo1:: URA3) was constructed from W303-1A as described above for the AS4 and AS13 exo1 derivatives. The wild-type diploid (W303) was constructed by crossing W303-1A with W303-1B while the exo1 diploid derivatives were constructed by crossing LSY496-10D with LSY496-20A (to generate LSY529a) and LSY496-20A with LSY625 (to generate LSY529b). The MSH5 gene was disrupted in the haploid W303-derived strains by the one-step replacement method using plasmid pNH190-11 (HollingSWORTH et al. 1995). The resulting strains, LSY810

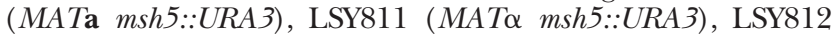
(MATa msh5::URA3 exo1::HIS3), and LSY813 (MATd msh5:: URA3 exo1::HIS3), were crossed to make the diploids LSY899 $($ LSY810 $\times$ LSY811) and LSY900 (LSY812 $\times$ LSY813). The genotypes of the mating-type tester strains were: MATa suc2-

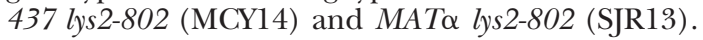

Statistical analysis: Statistical comparisons were done using the Instat 1.12 program for Macintosh (GraphPad Software). Chi-square test or Fisher's exact variant were used for comparisons. Results were considered statistically significant if $P<$ 0.05 .

\section{RESULTS AND DISCUSSION}

Reduced spore viability in diploids homozygous for the exo1 mutation: The diploid strains W303 (EXO1/ EXO1) and LSY529 (exo1/exo1) are isogenic except for the exo1 mutation. When these strains were sporulated after a limited number of generations (about 15) of vegetative growth following their construction, we found that the wild-type diploid had better spore viability than the homozygous exo1 diploid (94 vs. 87\%; Table 1); by a chi-square test this difference is highly significant $(P<$ $0.0001)$. Since strains with the exol mutation have elevated levels of spontaneous mutations (TishkofF et al. 1997), one interpretation of this result is that recessive lethal mutations accumulate in the diploid exo1 strain, leading to a loss of spore viability. By this interpretation, additional generations of vegetative growth of the diploid should lead to further reductions in spore viability. As shown in Table 1, although 60 generations of vegetative growth of LSY529 slightly reduced spore viability (from 87 to $79 \%$ ), a reduction was also observed in the wild-type diploid (from 94 to $89 \%$ ). We conclude, therefore, that the reduction in spore viability in the exo1 diploid is unlikely to reflect the accumulation of recessive lethal mutations.

Another potential cause of spore inviability is chromosome nondisjunction. A single nondisjunction event during meiosis I would be expected to result in two disomic spores and two spores missing one chromosome. Nondisjunction events involving two different chromosomes during meiosis I would be expected to result in either two double disome spores and two spores missing two chromosomes or four spores that are disomic for one chromosome and lack another. Thus, in strains with a moderate level of nondisjunction in meiosis I, one would expect to detect an elevation in classes of tetrads with two viable spores or with no viable spores. In contrast, classes of tetrads with three viable spores or one viable spore would not be substantially elevated as a consequence of meiosis I nondisjunction.

Two arguments are consistent with the conclusion that the exo1 mutations increase the rate of chromosome nondisjunction in meiosis I. First, when we compare the sum of the number of tetrads with two viable spores and no viable spores with the sum of the other classes of tetrads in exol and wild-type strains, we find a significant elevation in the exo1 strain $(P<0.0001)$. When we compare the sum of the number of tetrads with three viable spores and one viable spore with the sum of the other classes of tetrads, no significant elevation is ob- 
served in the exol strains $(P=0.6)$. The second argument is based on finding nonmating spores. If a nondisjunction event involves chromosome III (the location of the MAT locus), tetrads with two viable nonmating spores should be produced. We tested the mating phenotype of all of the spores derived from exo1 and wildtype strains shown in Table 1. As expected, all except six of the tetrads with four viable spores showed 4:4 segregation for MAT. The exceptions were gene conversion events observed in both strains. Of the tetrads with two viable spores, 11 of 93 from the LSY529 exo1 strains represented pairs of nonmating spores, indicative of chromosome III nondisjunction during meiosis I. No pairs of nonmating spores were observed for the wild-type strain. A second diploid version of LSY529 (LSY529b) was constructed in which one allele of EXO1 was disrupted with URA3 (exo1::URA3) and one allele with HIS3 (exo1::HIS3). As the EXO1 locus resides on chromosome $X V$, nondisjunction events involving chromosome $X V$ can be detected by monitoring the segregation of these two alleles. From the 48 2:2 spores (Table 1), there were 2 with $\mathrm{Ura}^{+} \mathrm{His}^{+}$spores, indicative of nondisjunction of chromosome $X V$. This number might be an underestimate, as the $\mathrm{Ura}^{+} \mathrm{His}^{+}$spores grow very poorly, presumably due to the extra copy of chromosome $X V$.

Chromosome nondisjunction during meiosis II would be expected to produce tetrads with three viable spores and one inviable spore. None of the tetrads with three viable spores contained a nonmating spore, indicating no detectable meiosis II nondisjunction in the wildtype or exo1 strains. In addition, as described above, the fraction of tetrads with three viable spores was not significantly affected by the exo1 mutation. In summary, we conclude that the spore inviability in exo1 strains reflects an increase in chromosome nondisjunction during meiosis I.

A number of other yeast mutants have been identified that decreased spore viability associated with reduced intergenic recombination including $m s h 4, m s h 5, m l h 1$, mlh3, zip1, zip2, and tam1 (Syм et al. 1993; Ross-MACDONAld and Roeder 1994; Hollingsworth et al. 1995; ChuA and Roeder 1997, 1998; WANG et al. 1999). The MSH4 and MSH5 genes are meiosis specific and encode homologues of bacterial MutS proteins (Ross-MACDONALD and Roeder 1994; Hollingsworth et al. 1995). Unlike other MutS homologues, $m s h 4$ and $m s h 5$ mutants show no apparent defect in mismatch correction during mitosis or meiosis. Instead, the proteins are proposed to form a heterodimer that binds to Holliday junctions and promotes resolution of recombination intermediates to form crossover products (Pochart et al. 1997). The meiotic phenotype of exol mutants resembles msh 4 and $m s h 5$ mutants, except that the defect in spore viability in exo1 strains is less severe. $m l h 1$ mutants also show a decrease in intergenic recombination and spore viability and epistasis studies indicate that MLH1 functions in the same pathway as MSH4 and MSH5 (HunTer and BORTs 1997). To determine whether EXO1 functions in the same pathway as MSH5, double mutants were generated and examined for spore viability. In the W303 genetic background, a strain homozygous for the msh 5 mutation (LSY899) had substantially reduced spore viability (55\% spore viability from 230 tetrads dissected). In the diploid strain LSY900, homozygous for both $m s h 5$ and exo1, the spore viability was not significantly different from the msh 5 homozygote (53\% from 637 tetrads dissected). A strain homozygous for exo1 and msh 4 mutations showed similar spore viability ( $47 \%$ from 200 tetrads dissected).

We also examined the effects of the exo1 and msh 5 mutations on spore viability in a different genetic background [crosses of AS4- and AS13-derived haploid strains (Stapleton and Petes 1991)]. In the EXO1/ EXO1 diploid strain DTK347, we observed $85 \%$ spore viability. In the exo1/exo1 strains DTK308, DTK332, DTK333, and DTK335 (differing in alleles at the HIS4 locus), the average spore viability was $78 \%$, a significant $(P<$ $0.0001)$ reduction from that observed for the wild-type strain. An $m s h 5 / m s h 5$ strain (DTK498) had a spore viability of $80 \%$, also a significant reduction $(P=0.0001)$ compared to wild type. The average spore viability in a strain homozygous for deletions in both exo1 and msh 5 (DTK505) was 67\%, a significant reduction compared to wild-type, msh5, and exo1 strains $(P<0.0001)$, but above the level expected for independent pathways $(80 \% \times$ $78 \%=62.4 \%)$. Thus, in two different genetic backgrounds, spore viability is reduced by mutations in EXO1 and MSH5, and analysis of spore viability in the double mutant indicates that Exo1p and Msh5p function in the same or overlapping pathways for chromosome disjunction. This contrasts with the results obtained with exo1 msh4 homozygous diploids in two other strain backgrounds, in which synergistic decreases in spore viability were observed (KHAZANEHDARI and BorTs 2000; TsuBOUCHI and OgaWA 2000).

Reduced spore viability associated with elevated levels of meiosis I chromosome nondisjunction is often a consequence of decreased meiotic crossing over (RoEDER 1997). Consequently, as described below, we examined the effects of the exo1 mutation on the frequency of reciprocal and nonreciprocal meiotic recombination.

Reduced meiotic crossing over in strains homozygous for the exo1 mutation: We examined meiotic recombination in four pairs of diploid wild type and exo1 mutant strains (Figure 1). Each diploid was constructed from AS4- and AS13-derived haploid strains and was heterozygous for mutations at the HIS4 locus (located on chromosome III), the linked (centromere-proximal) LEU2 locus, and the centromere-linked TRP1 locus. We monitored crossovers between HIS4 and LEU2 and between $L E U 2$ and CEN3, as well as the frequency of aberrant segregation at the HIS4 locus. As will be discussed in more detail below, the four pairs of strains differed in 

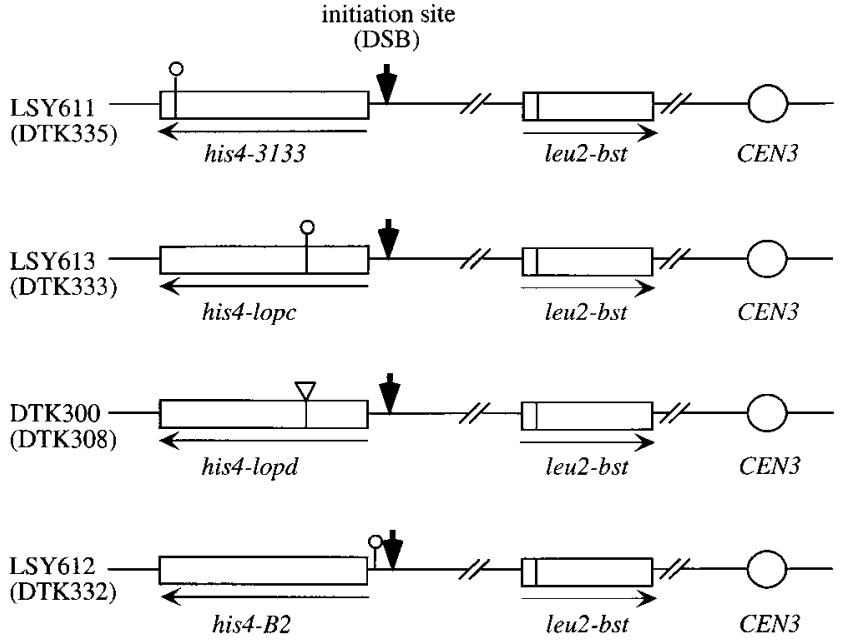

FIGURE 1.-Location of markers within the his 4 gene. Haploid strains LSY611, LSY613, DTK300, and LSY612 were made by one-step gene replacement of strains PD98, DNY25, DNY24, and DNY16, respectively, using a PCR-generated DNA fragment designed to disrupt the EXO1 gene. The vertical arrow indicates the position of the recombination initiation site upstream of the HIS4 gene; the horizontal arrows indicate the direction of transcription. The lollipops indicate the position of palindromic insertions within his 4 . The inverted triangle represents the nonpalindromic insertion within his4. Each haploid was crossed to the $\mathrm{His}^{+}$strain DTK299 to create the diploids DTK308, DTK332, DTK333, and DTK335. The corresponding diploid strain number is given in parentheses.

the nature and/or position of the heterozygous his 4 mutations.

As shown in Table 2, for all four pairs of strains the map distances between HIS4 and LEU2 and between
LEU2 and the centromere were reduced by the exo1 mutation. This reduction was statistically significant $(P<0.03)$ for both genetic intervals in every strain pair, except for the HIS4-LEU2 interval in strains PD99 and DTK335 $(P=0.2)$. We conclude, therefore, that the exo1 mutation reduces but does not eliminate crossing over. It is likely that this reduction is responsible for the elevation in chromosome nondisjunction and the reduced spore viability observed in the exo1 strains.

The relationship between EXO1-dependent crossing over and the previously defined pathway for crossing over that involves MLH1, MLH3, MSH4, and MSH5 (described above) was determined by examining the level of intergenic recombination in $m s h 5$ and $m s h 5$ exo 1 strains (Table 2). Deletion of $M S H 5$ reduced the map distance between HIS4 and LEU2 to the same degree as a deletion of EXO1 [from $39 \mathrm{cM}$ to 24 (msh5) and $22 \mathrm{cM}$ (exo1), respectively]. The HIS4-LEU2 map distance in the double mutant $(19 \mathrm{cM})$ was not statistically different than the distances in the single mutants, indicating that the two gene products function in the same pathway for intergenic recombination.

Effect of the exo1 mutation on aberrant segregation at the HIS4 and ARG4 loci: Three of the pairs of AS4 $\times$ AS13 strains used in this study are heterozygous for palindromic insertions; one member of each pair was wild type and one member was homozygous for exol. The location of these insertions relative to the HIS4 initiating codon are: -50 (his4-B2), +467 (his4-lopc), and +2327 (his4-3133) (Figure 1). The fourth pair of strains (DNY27 and DTK308) was heterozygous for a nonpalindromic insertion at the same position in HIS4 as the his4-lopc insertion. Tetrads from each strain were

TABLE 2

Map distances between various loci in EXO1 or exo1 strains

\begin{tabular}{|c|c|c|c|c|c|c|c|c|c|c|c|}
\hline \multirow[b]{2}{*}{ Strain } & \multirow[b]{2}{*}{ HIS4 } & \multirow[b]{2}{*}{ EXO1 } & \multicolumn{5}{|c|}{ HIS4-LEU2 interval } & \multicolumn{4}{|c|}{$L E U 2-C E N 3$ interval } \\
\hline & & & PD & NPD & $\mathrm{T}$ & $\mathrm{cM}$ & $\%$ of $\mathrm{WT}$ & FDS & SDS & $\mathrm{cM}$ & $\%$ of WT \\
\hline $\mathrm{DNY}_{1} 8^{a}$ & his4-B2/+ & $+/+$ & 155 & 10 & 124 & 32 & 100 & 280 & 75 & 11 & 100 \\
\hline DTK332 & his4-B2/+ & $-/-$ & 113 & 4 & 37 & 20 & 63 & 163 & 17 & 5 & 45 \\
\hline DNY26 $6^{b}$ & his4-lopc/+ & $+/+$ & 191 & 8 & 202 & 31 & 100 & 388 & 91 & 10 & 100 \\
\hline DTK333 & his4-lopc/+ & $-/-$ & 109 & 1 & 53 & 18 & 58 & 170 & 13 & 4 & 40 \\
\hline $\mathrm{PD}^{c}{ }^{c}$ & his4-3133/+ & $+/+$ & 202 & 12 & 141 & 30 & 100 & 320 & 88 & 11 & 100 \\
\hline DTK335 & his4-3133/+ & $-/-$ & 112 & 2 & 67 & 22 & 73 & 187 & 13 & 3 & 27 \\
\hline $\mathrm{DNY} 27^{d}$ & his4-lopd/+ & $+/+$ & 130 & 15 & 108 & 39 & 100 & 289 & 65 & 9 & 100 \\
\hline DTK308 & his4-lopd/+ & $-/-$ & 156 & 4 & 79 & 22 & 56 & 261 & 21 & 4 & 44 \\
\hline DTK498 & his4-lopd/ + & $+/+m s h 5 / m s h 5$ & 143 & 5 & 82 & 24 & 61 & 249 & 32 & 6 & 67 \\
\hline DTK505 & his4-lopd/+ & $-/-m \operatorname{sh} 5 / m s h 5$ & 124 & 1 & 69 & 19 & 49 & 216 & 43 & 8 & 89 \\
\hline
\end{tabular}

All strains are diploids that are isogenic except for the differing heterozygous HIS4 allele and the presence or absence of EXO1 or MSH5. The location of each of the HIS4 alleles is depicted in Figure 1. PD, parental ditype; NPD, nonparental ditype; T, tetratype; FDS, first division segregation; SDS, second division segregation; cM, centimorgans; WT, wild type. The number of tetrads of each segregation class is listed in the appropriate column.

${ }^{a}$ From NAG et al. (1989).

${ }^{b}$ From FAN et al. (1995).

${ }^{c}$ Rescored from original data (Detloff and Petes 1992).

${ }^{d}$ From Kirkpatrick and Petes (1997). 
dissected and the level of aberrant segregation at the HIS4 locus was monitored (Table 3). All of the strains are also heterozygous for a mutation in ARG4; the level of aberrant segregation of this marker was also determined.

The level of aberrant segregation for strains containing a palindromic insertion at HIS4 is only slightly affected by the exo1 mutation (Table 3); the effects on aberrant segregation are considerably smaller than on crossing over. The exo1 mutation has its strongest effect on the level of aberrant segregation for the strain with the nonpalindromic insertion (compare DNY27 and DTK308); an interpretation of this result will be given below. The exo1 mutation does not significantly reduce the frequency of aberrant segregation of the palindromic insertion near the 3' end of HIS4 (comparison of PD98 and DTK335). Since most of the heteroduplexes located at the $3^{\prime}$ end of HIS4 reflect processing of a DSB located at the $5^{\prime}$ end of HIS4, this result indicates that Exo1p is not required for producing the singlestranded DNA "tail" required for heteroduplex formation.

The effect of deletion of exo 1 on aberrant segregation of a heterozgyous allele at the $A R G 4$ locus contrasts with the effect on aberrant segregation at HIS4. In the wildtype strain DNY27, 44 out of 359 tetrads exhibited aberrant segregation at ARG4 (24 6:2 events and 20 2:6 events). In the exo1 strains (DTK308, DTK332, DTK333, and DTK335) there were 37 tetrads of a total of 870 that exhibited aberrant segregation of the ARG4 marker (23 6:2 events and 14 2:6 events). This decrease in the level of aberrant segregation, from $12 \%$ in the wild type to $4 \%$ in the exo1 mutant strains, is highly significant $(P<0.0001)$. All events in both wild-type and exo1 strains were gene conversions; no postmeiotic segregation (PMS) tetrads were detected. Removal of Exolp affects the observed level of aberrant segregation differently at HIS4 and ARG4. At HIS4, the data indicate that the level of aberrant segregation is not significantly affected by loss of Exo1p, whereas the level of aberrant segregation observed at the ARG4 locus is reduced. Locus-specific effects resulting from the loss of Exolp have been reported previously (KHAZANEHDARI and BorTS 2000). This study on the effects of a deletion of EXO1 on the aberrant segregation rate of a number of twoor four-base insertions into HIS4 and LEU2 demonstrated that the HIS4 alleles, but not the LEU2 alleles, showed reduced aberrant segregation when EXO1 was deleted (Khazanehdari and BorTs 2000).

Effect of the exo1 mutation on patterns of aberrant segregation at the HIS4 locus: In strains heterozygous for a single marker, most tetrads segregate 4:4 (nomenclature used for eight-spored fungi). As discussed in the Introduction, the most common aberrant segregation types are of two classes, gene conversion events $(6: 2$ or 2:6 segregations) or postmeiotic segregation events (5:3 or 3:5 segregation). PMS tetrads reflect asymmetric het- eroduplex formation between wild-type and mutant alleles and failure to correct the resulting DNA mismatch. The efficiency of repair of a mismatch is reflected by the fraction of the aberrant segregation events that are PMS. In S. cerevisiae, single base mismatches (excluding $\mathrm{C} / \mathrm{C}$ ) and mismatches resulting from nonpalindromic insertions are efficiently repaired (low level of PMS compared to gene conversion), whereas $\mathrm{C} / \mathrm{C}$ mismatches and mismatches resulting from small palindromic insertions are inefficiently repaired (Petes et al. 1991).

In the AS4 $\times$ AS13 genetic background, the HIS4 locus has a very high level of aberrant segregation (NAG et al. 1989). Most aberrant segregation events at the HIS4 locus reflect an initiating double-strand DNA break near the $5^{\prime}$ end of the gene (FAN et al. 1995), followed by heteroduplex formation that often extends from the break to the 3' end of HIS4 (Porter et al. 1993). One argument supporting the conclusion that heteroduplex formation usually involves the entire HIS4 coding region is that the level of aberrant segregation for markers leading to inefficiently repaired DNA mismatches at the $5^{\prime}$ and $3^{\prime}$ ends of the HIS4 gene is similar (Detloff et al. 1992). For most of the exo1 strains, there was a significant increase in the number of PMS events at the HIS4 locus compared with the wild-type diploids, confirming the role of Exolp in mismatch repair (Table 3). Both the his4-B2 and his4-lopc strains showed a significant $(P=$ 0.04 and 0.03 , respectively) increase in the number of PMS tetrads when EXO1 was deleted. The greatest increase $(P=0.007)$ in such tetrads was seen in the strain DTK308 containing the well-repaired his4-lopd allele, which generates a 26-base loop when contained in a heteroduplex tract. We previously demonstrated (KIRKPatrick and Petes 1997) that correction of this loop was also dependent on the products of the RAD1 and MSH2 genes, although deletion of either of these two genes has a greater effect on correction of his4-lopd mismatches than a deletion of EXO1. In all pairs of strains, the exo1 member of the pair had a reduced frequency of gene conversion relative to the wild-type member (Table 3). For example, in strains heterozygous for the nonpalindromic his4-lopd insertion, the gene conversion frequency was reduced from 26 to $14 \%$ by the exo1 mutation, and the PMS frequency was elevated from 4.3 to $7 \%$. These results are the first demonstration of a role for Exolp in DNA mismatch repair during meiosis; other studies did not show an increase in the frequency of PMS of heteroalleles when EXO1 was deleted (Khazanehdari and Borts 2000; Tsubouchi and Ogawa 2000). Also, in our study no increase in PMS was detected at the ARG4 locus, as described above. The alleles that show no increase in PMS are those that will generate a small mismatch (one to four bases) when present in heteroduplex, while the alleles that have a significant increase in PMS are those that will generate a large mismatch (26 to 36 bases) in heteroduplex DNA. 


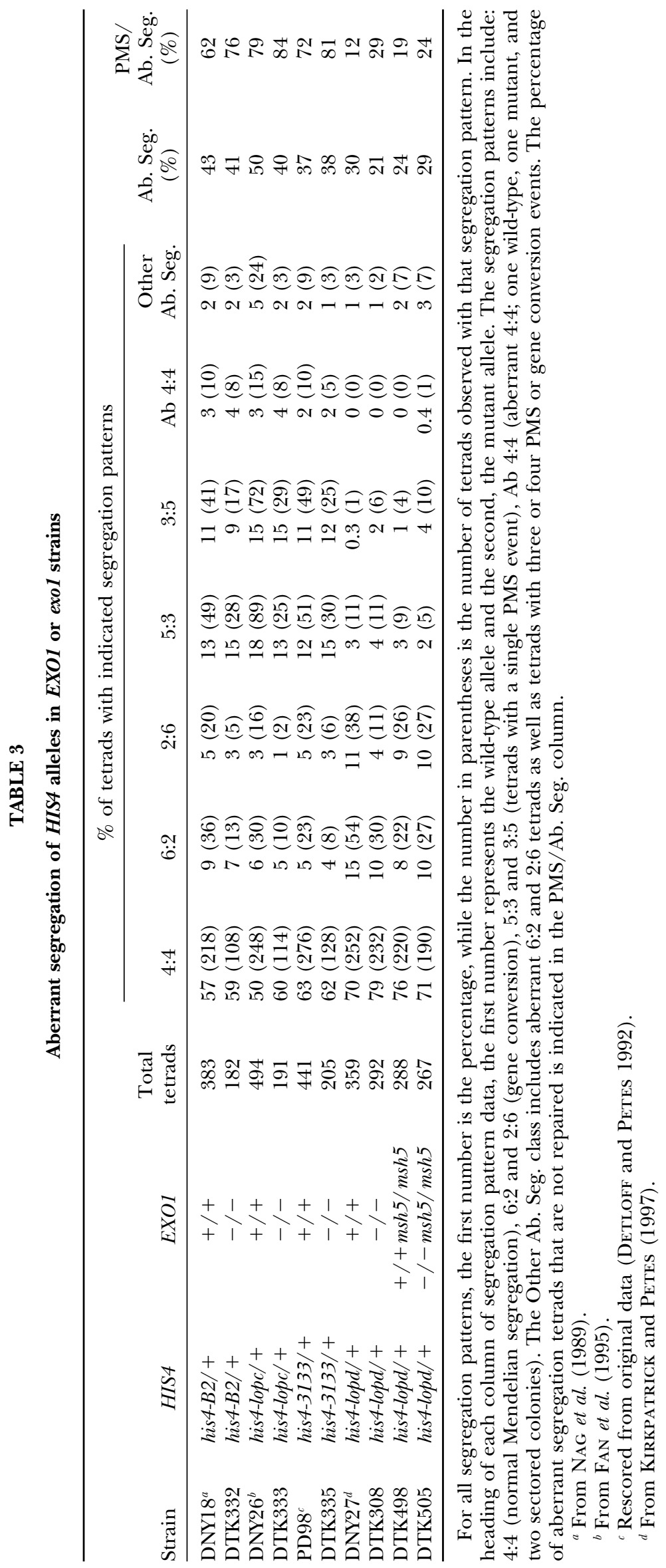


We propose that Exolp may have a role specifically in the repair of large mismatches.

Conclusions: In conclusion, we find that Exolp promotes meiotic crossing over, ensuring proper disjunction of chromosomes during meiosis I. This function involves a previously identified pathway involving the MSH4, MSH5, MLH1, and MLH3 gene products (RossMacdonald and Roeder 1994; Hollingsworth et al. 1995; Hunter and Borts 1997; WANG et al. 1999), as the level of crossovers in strains lacking both Exolp and Msh5p is higher than expected if the two proteins work in distinct pathways. Loss of Exolp also leads to a decrease in spore viability. This decrease is not due to the accumulation of mutations during mitotic growth and is partially overlapping with the decrease in spore viability seen in strains lacking Msh5p. Furthermore, the length of the heteroduplex tract at the HIS4 locus does not appear to be reduced in exo1 mutant strains, indicating that the Exolp deoxyribonuclease does not participate in generating long 3 ' single-stranded tailed molecules following double-strand break generation during meiotic recombination at HIS4. It is possible that the nuclease function of Exolp acts postsynaptically to direct resolution of recombination intermediates instead of the predicted presynaptic function. Alternatively, the EXO1 protein could participate in generating a small amount of single-stranded DNA, after which another protein or protein complex continues the process of generating the long 3 ' single-stranded tailed molecules present at HIS4. In addition, we find that the exo1 mutation affects the frequency of aberrant segregation in a manner that suggests that Exolp has a minor role in the repair of DNA mismatches at HIS4. Exolp is involved in the repair of a 26-bp loop mismatch in heteroduplex DNA, although to a lesser extent than several other proteins (Rad1p and Msh2p) known to act on DNA loops of this size (Kirkpatrick and Petes 1997). Finally, there are locus-specific effects to the loss of Exolp, as the level of aberrant segregation at ARG4 is significantly lowered by loss of Exolp, unlike the situation at HIS4. Also in contrast to the data from the HIS4 locus, repair of mismatches at $A R G 4$ is not affected by the exo 1 mutation, indicating that at $A R G 4$ initiation, rather than repair, is primarily affected by the exo1 mutation.

This work was supported by Public Health Service grants from the National Institutes of Health GM41784 (L.S.S.), 2 T32 CA09503 (J.R.F.), and GM24110 (T.D.P.). D.T.K. is a Special Fellow of the Leukemia and Lymphoma Society.

\section{LITERATURE CITED}

Cao, L., E. Alani and N. Kleckner, 1990 A pathway for generation and processing of double-strand breaks during meiotic recombination in S. cerevisiae. Cell 61: 1089-1101.

Chua, P. R., and G. S. Roeder, 1997 Tam1, a telomere-associated meiotic protein, functions in chromosome synapsis and crossover interference. Genes Dev. 11: 1786-1800.

Chua, P. R., and G. S. Roeder, 1998 Zip2, a meiosis-specific protein required for the initiation of chromosome synapsis. Cell 93: 349 359.

Detloff, P., and T. D. Petes, 1992 Measurements of excision repair tracts formed during meiotic recombination in Saccharomyces cerevisiae. Mol. Cell. Biol. 12: 1805-1814.

Detloff, P., M. A. White and T. D. Petes, 1992 Analysis of a gene conversion gradient at the HIS4 locus in Saccharomyces cerevisiae. Genetics 132: 113-123.

Fan, Q., F. Xu and T. D. Petes, 1995 Meiosis-specific double-strand DNA breaks at the HIS4 recombination hot spot in the yeast Saccharomyces cerevisiae: control in cis and trans. Mol. Cell. Biol. 15: $1679-1688$.

Fiorentini, P., K. N. Huang, D. X. Tishkoff, R. D. Kolodner and L. S. Symington, 1997 Exonuclease I of Saccharomyces cerevisiae functions in mitotic recombination in vivo and in vitro. Mol. Cell. Biol. 17: 2764-2773.

Furuse, M., Y. Nagase, H. Tsubouchi, K. Murakami-Murofushi, T. Shibata et al., 1998 Distinct roles of two separable in vitro activities of yeast Mre11 in mitotic and meiotic recombination. EMBO J. 17: 6412-6425.

Hollingsworth, N. M., L. Ponte and C. Halsey, 1995 MSH5, a novel MutS homolog, facilitates meiotic reciprocal recombination between homologs in Saccharomyces cerevisiae but not mismatch repair. Genes Dev. 9: 1728-1739.

Huang, K. N., and L. S. Symington, 1993 A 5'-3' exonuclease from Saccharomyces cerevisiae is required for in vitro recombination between linear DNA molecules with overlapping homology. Mol. Cell. Biol. 13: 3125-3134.

Hunter, N., and R. H. BorTs, 1997 Mlh1 is unique among mismatch repair proteins in its ability to promote crossing-over during meiosis. Genes Dev. 11: 1573-1582.

Keeney, S., C. N. Giroux and N. Kleckner, 1997 Meiosis-specific DNA double-strand breaks are catalyzed by Spo11, a member of a widely conserved protein family. Cell 88: 375-384.

Khazanehdari, K. A., and R. H. Borts, $2000 \quad$ EXO1 and MSH4 differentially affect crossing-over and segregation. Chromosoma 109: $94-102$.

Kirkpatrick, D. T., and T. D. Petes, 1997 Repair of DNA loops involves DNA-mismatch and nucleotide-excision repair proteins. Nature 387: 929-931.

Kirkpatrick, D. T., Y. H. Wang, M. Dominska, J. D. Griffith and T. D. Petes, 1999 Control of meiotic recombination and gene expression in yeast by a simple repetitive DNA sequence that excludes nucleosomes. Mol. Cell. Biol. 19: 7661-7671.

Moreau, S., J. R. Ferguson and L. S. Symington, 1999 The nuclease activity of Mre11 is required for meiosis, but not for mating type switching, end joining, or telomere maintenance. Mol. Cell. Biol. 19: 556-566.

NAG, D. K., M. A. White and T. D. Petes, 1989 Palindromic sequences in heteroduplex DNA inhibit mismatch repair in yeast. Nature 340: 318-320.

Petes, T. D., R. E. Malone and L. S. Symington, 1991 Recombination in Yeast. Cold Spring Harbor Laboratory Press, Cold Spring Harbor, NY.

Pochart, P., D. Woltering and N. M. Hollingsworth, 1997 Conserved properties between functionally distinct MutS homologs in yeast. J. Biol. Chem. 272: 30345-30349.

Porter, S. E., M. A. White and T. D. Petes, 1993 Genetic evidence that the meiotic recombination hotspot at the HIS4 locus of Saccharomyces cerevisiae does not represent a site for a symmetrically processed double-strand break. Genetics 134: 5-19.

Roeder, G. S., 1997 Meiotic chromosomes: it takes two to tango. Genes Dev. 11: 2600-2621.

Ross-Macdonald, P., and G. S. Roeder, 1994 Mutation of a meiosisspecific MutS homolog decreases crossing over but not mismatch correction. Cell 79: 1069-1080.

Sherman, F., G. Fink and J. Hicks, 1986 Methods in Yeast Genetics. Cold Spring Harbor Laboratory Press, Cold Spring Harbor, NY.

Sikorski, R. S., and P. Hieter, 1989 A system of shuttle vectors and yeast host strains designed for efficient manipulation of DNA in Saccharomyces cerevisiae. Genetics 122: 19-27.

Stapleton, A., and T. D. Petes, 1991 The Tn3 beta-lactamase gene acts as a hotspot for meiotic recombination in yeast. Genetics 127: $39-51$.

Sun, H., D. Treco and J. Szostak, 1991 Extensive 3'-overhang, single-stranded DNA associated with the meiosis-specific double- 
strand breaks at the $A R G 4$ recombination initiation site. Cell 64: $1155-1161$.

Sym, M., J. A. ENGebrecht and G. S. Roeder, 1993 ZIP1 is a synaptonemal complex protein required for meiotic chromosome synapsis. Cell 72: 365-378.

Szankasi, P., and G. R. Smith, 1992 A DNA exonuclease induced during meiosis of Schizosaccharomyces pombe. J. Biol. Chem. 267: 3014-3023.

Szankasi, P., and G. R. Smith, 1995 A role for exonuclease I from $S$. pombe in mutation avoidance and mismatch correction. Science 267: 1166-1169.

Tishkoff, D. X., A. L. Boerger, P. Bertrand, N. Filosi, G. M. Gaida et al., 1997 Identification and characterization of Saccharomyces cerevisiae EXO1, a gene encoding an exonuclease that interacts with MSH2. Proc. Natl. Acad. Sci. USA 94: 7487-7492.

Trujillo, K. M., S.-S. F. Yuan, E. Y.-H. Lee and P. Sung, 1998 Nuclease activities in a complex of human recombination and DNA repair factors Rad50, Mre11, and p95. J. Biol. Chem. 273: 21447-21450.
Tsubouchi, H., and H. Ogawa, 2000 Exol roles for repair of DNA double-strand breaks and meiotic crossing over in Saccharomyces cerevisiae. Mol. Biol. Cell 11: 2221-2233.

Usui, T., T. Оhta, H. Oshiumi, J. Tomizawa, H. Ogawa et al., 1998 Complex formation and functional versatility of Mre11 of budding yeast in recombination. Cell 95: 705-716.

Wach, A., A. Brachat, R. Pohlmann and P. Philippsen, 1994 New heterologous modules for classical or PCR-based gene disruptions in Saccharomyces cerevisiae. Yeast 10: 1793-1808.

WANG, T. F., N. KLECKNER and N. HunTer, 1999 Functional specificity of MutL homologs in yeast: evidence for three Mlh1-based heterocomplexes with distinct roles during meiosis in recombination and mismatch correction. Proc. Natl. Acad. Sci. USA 96: 13914-13919.

Communicating editor: M. LichTEN 